\title{
FUNCTIONAL CHARACTERIZATION OF RECOMBINANT INTERLEUKIN (IL)-17A/F1 IN THE JAPANESE PUFFERFISH (TAKIFUGU RUBRIPES)
}

\author{
Jun-ichi Hikima ${ }^{1}$, Koshin Mihara ${ }^{1}$, Shun Makawa ${ }^{2}$, Han-Ching Wang ${ }^{2}$, Takashi Aoki ${ }^{2}$, \\ Tomoya Kono ${ }^{1}$, Masahiro Sakai ${ }^{1 *}$ \\ ${ }^{1}$ Department of Biochemistry and Applied Biosciences, Faculty of Agriculture, University of \\ Miyazaki, Miyazaki 889-2192, Japan \\ ${ }^{2}$ Institute of Biotechnology, College of Biosciences and Biotechnology, National Cheng Kung \\ University, Tainan 701, Taiwan ROC
}

\begin{abstract}
In mammals, interleukin (IL)-17A and IL-17F are hallmark inflammatory cytokines, which are expressed by Th17 cells. In contrast, teleost IL-17A and IL-17F homologs named as IL-17A/F has been identified, and its functional aspect is poorly understood. Here, the recombinant protein of the Japanese pufferfish, Fugu (Takifugu rubripes) IL-17A/F1 was produced and purified using mammalian cell line expression system where the protein folding was achieved correctly. The recombinant $(r)$ IL-17A/F1 was added into head kidney (HK) and intestinal endothelial (IE) cells prepared from Fugu to stimulate the immune regulations, and then phagocytic activity, superoxide anion production by NBT assay, and cytokine gene expressions in the treated HK and IE cells at 0, 3, 6, 12, 24 and 48 hours post-stimulation (hps) were conducted. The rIL-17A/F1-treated both HK and IE cells showed an increased phagocytic activity at $24 \mathrm{hps}$. Superoxide anion production increased significantly at $48 \mathrm{hps}$ in the HK cells and also increased moderately at $48 \mathrm{hps}$ in the IE cells. Moreover, the $r \mathrm{IL}-17 \mathrm{~A} / \mathrm{F} 1$ displayed an ability to enhance expression of pro-inflammatory cytokine mRNAs, such as IL-1 $\beta$, TNF- $\alpha$ and IL-6 in Fugu. The expression of IL-1 $\beta$, TNF- $\alpha$ and IL-6 genes was significantly up-regulated during 3 to $12 \mathrm{hps}$. These bioactivity and gene expression results suggest that Fugu IL-17A/F1 probably plays an important role in promoting inflammatory and host innate immune responses as a pro-inflammatory cytokine in fish.
\end{abstract}

\section{KEYWORDS}

IL-17A/F, pro-inflammatory cytokine, phagocytic activity, gene expression, Japanese pufferfish

*Corresponding author. Tel/Fax: +81-985-58-7219

E-mail address: m.sakai@cc.miyazaki-u.ac.jp 